\title{
An Effective M-ary Spread Spectrum Communication Scheme
}

\author{
Yuexuan Liu, Xiaohui Li, Peng Gao, Hongdong Li and Rui Dong
}

Training Department, PLA Communication Training Base of GSD, Zhangjiakou, Hebei, China (yuexuanliu@163.com)

\begin{abstract}
A new M-ary spread spectrum(SS) hybrid communication system is proposed. The system adopts two orthogonal branches to transport information. In each branch, double M-ary biorthogonal spread spectrum modulation is adopted at first, then every SS code is treated as original code to adopt CPSK modulation, and the unattached PN code is chosen to achieve system synchronization. The proposed scheme is easy to achieve synchronization, and is effective in improving the bandwidth efficiency and anti-intercept capability. Computer simulation show the validity of the proposed scheme under additional white gauss noise channel models.
\end{abstract}

Keywords-M-ary spread spectrum, dual orthogonal spread spectrum, code phase shifting keying (CPSK), circular correlation

\section{一种高效多进制扩频通信方案}

刘跃宣 李晓辉高鹏 李洪冬 董 锐

总参通信训练基地训练部，张家口，河北，中国

摘 要 提出了一种新的多进制扩频通信方案。该系统采用两条正交支路传输信息, 在每条支路上, 首先采用 M-ary 双正交扩频 调制, 再以每一个扩频码为原型码进行 CPSK 调制, 并选择专用的 PN 码进行同步。该方案易于实现同步, 同时具有较高的频带利用 率和更强的抗截获性能。计算机仿真证明了其有效性。

关键词 多进制扩频，双正交扩频，CPSK，循环相关

\section{1. 引言}

直接序列扩频通信因具有较强的抗干扰、抗截获、抗 多径性能, 广泛应用于军事保密通信、电子对抗、雷达、 导航、测距、定位等领域, 但是其频带利用率较低。多进 制扩频通信 (或称为 $\mathrm{M}$ 元正交扩频) 与传统的直扩系统相 比,在相同的带宽条件下可以获得更高的信息传输速率或 扩频处理增益，大大提高了频带利用率，且其频谱更接近 于白噪声, 从而具有更强的抗截获性和信息保密性。

文献[1][2]选用 $M$ 个相互正交 (或近似正交) 的伪噪声 码 (Pseudo Noise Code, PN 码) 构成扩频码集, 利用一个 扩频码传输 $\log _{2} M$ 比特信息, 称为正交扩频。在正交扩频 的基础上进行 BPSK 调制, 称为双正交扩频[3], 这样可以 多传输 1 bit 信息, 或节省一半的扩频码数量。这种多元扩 频方式所需扩频码的条数与每符号所包含的比特数成指数 关系, 随着所需扩频码条数的增加, 选码的难度相应增加, 同时扩频码的捕获和跟踪也变得困难。

文献[4][5]利用一条原型 PN 码的循环移位码构成扩频

(C) 2014. The authors - Published by Atlantis Press
码集，利用扩频码相位携带信息，可以实现与正交扩频同 样的效果, 且大大减少了对扩频码的需求, 称为码相位循 环移位调制(code phase shifting keying, CPSK)扩频, 或者循 环码移键控(cyclic code shift keying, CCSK)扩频。但它需 要额外提供扩频码同步信息。CPSK 扩频系统的扩频码同 步主要有同步信道法和帧头法。文献[6]在正交信道的两个 支路分别采用 CPSK 扩频和 BPSK-DS 扩频调制, 通过两者 的互补特点, 实现系统同步, 并且提高了频带利用率; 文 献[7]在正交信道的两个支路上分别进行 CPSK 扩频, 并用 帧头法进行同步，进一步提高了频带利用率。

本文提出了一种混合多进制扩频通信方案：在双极性 正交扩频的基础上, 再以每一个扩频码为原型码进行 CPSK 调制, 并选择独立于信息码的同步码, 用帧头法进行同步。 与传统的正交扩频和 CPSK 扩频相比, 该扩频通信方案进 一步提高了信息传输速率，且具有优良的同步性能。

\section{2. 混合多进制扩频通信方案}

在发射端, 将信源产生的二进制数据流进行串并转换, 
分成 $I 、 Q$ 支路。每路每次传输 $k=m+n$ 比特信息。首先对 前 $m$ 比特信息数据进行 $U=2^{m}$ 进制双正交扩频：选取 $U / 2$ 个相互正交 (或近似正交) 的 $\mathrm{PN}$ 码 $P N_{1}, P N_{2} \ldots P N_{U / 2}$, 令扩 频码 $C_{(1,0)}=P N_{1}, C_{(2,0)}=P N_{2} \ldots C_{(U / 2,0)}=P N_{U / 2}, C_{(U / 2+1,0)}=-P N_{l}$, $C_{(U / 2+2,0)}=-P N_{2} \ldots C_{(U, 0)}=-P N_{U / 2}$, 构成扩频码集 $\left\{C_{(u, 0)} \mid u=1\right.$, $2 \ldots U\}$, 其中第二个下标 0 表示扩频码循环移位值为零。根 据 $m$ 比特信息数据从该码集中选择一个扩频码 $C_{(u, 0)}$ 与之对 应。再对后 $n$ 比特信息数据进行 $V=2^{n}$ 进制的 CPSK 调制: 以上述选定的扩频码 $C_{(u, 0)}$ 作为原型码进行循环移位, 共产 生包括原型码在内的 $V$ 个循环移位码构成 CPSK 扩频码集 $\left\{C_{(u, v)} \mid v=1,2 \ldots V\right\}$, 根据后 $n$ 比特信息数据从该码集中选择 一个扩频码 $C_{(u, v)}$ 与之对应。这样, 信道上传输的每一个扩 频码携带 $k=m+n$ 比特信息, 需要扩频码共有 $M=2^{m+n}=U \times V$ 个不同状态。然后组帧, 在帧头加入同步码: 选择与所有 信息码正交的伪噪声码 $P N_{s y n}$ 作为同步码, 在正交信道的两 个支路同时传递以提取同步信息。最后信号以正交调制的 方式发射出去。可见, 本系统需要 $U / 2+1$ 条 PN 码。发射 端系统原理框图如图 1 所示。

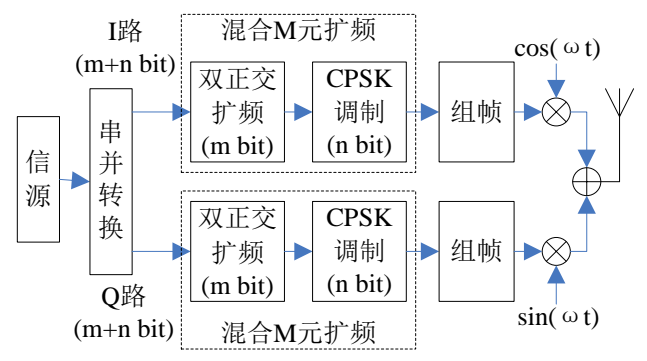

图 1 发射端系统原理框图

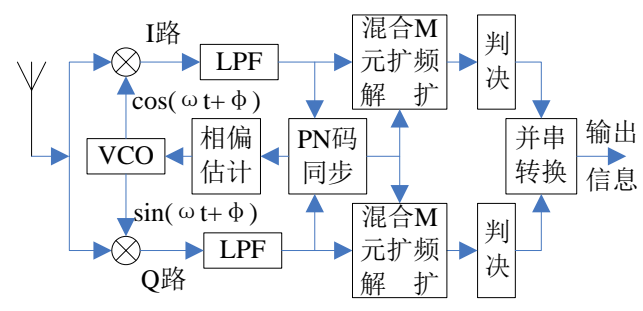

图 2 接收端系统原理框图

在接收端, 首先进行正交下变频, 把接收到的射频信 号变换为 $I 、 Q$ 两个支路的基带信号。 $\mathrm{PN}$ 码同步模块用非 相干解扩实现码同步, 并进行载波相偏估计 (本文未考虑 载波频偏), 载波相偏信息反馈到压控振荡器 (Voltage Controlled Oscillator, VCO）实现载波同步。系统同步后， 在每个支路用基于变换域处理的循环相关器 [4][5]进行混 合 $\mathrm{M}$ 元扩频的解扩, 并进行判决(算法详见本文第 3 部分), 最后进行并串转换, 得到原始的二进制数据流。接收端系
统原理框图如图 2 所示。

\section{3. 同步算法和性能}

同步是通信系统可靠工作的前提, 同步系统的性能对 整个系统的性能有非常重要的影响。单独占用一个扩频码 用来实现高性能的同步是值得的。在扩频系统中, 同步包 括载波同步和扩频码同步。其中载波同步包括载波频率同 步和相位同步[8]。由于对载波频偏的估计和纠正的算法较 多[9][10], 本文对载波频偏的影响不再分析, 认为无载波频 偏。

\section{1 同步原理}

在接收端采用非相关解扩实现 PN 码同步, 系统原理 框图如图 3 所示。由于用于同步的扩频码独立于传递信息 的扩频码, 所以同步码与所有的信息码及其循环移位码都 正交。当本地同步码与发送的同步码同相时, 同步系统输 出峰值, 理想情况下其他时刻同步系统输出均为零。所以 同步系统峰值出现时刻即为系统同步时刻。

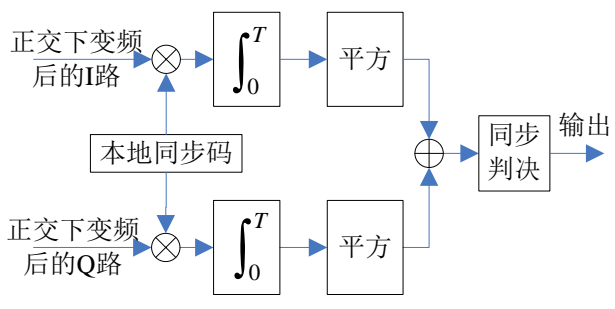

图 3 同步系统原理框图

为便于分析, 暂时忽略信道噪声的影响。设帧长为 $N$ 个扩频码长度, 即每传 $(N-1)$ 条信息码就插入一个同步码。 扩频码的周期为 $T$, 则帧周期为 $N T$ 。发送端信号为:

$$
s(t)=P N_{I}(t) \cos (2 \pi f t)+P N_{Q}(t) \sin (2 \pi f t)
$$

其中, $f$ 为载波频率, $P N_{I}(t) 、 P N_{Q}(t)$ 表示发送端 $I$ 路和 $Q$ 路的扩频码, 当发送数据信息时, $P N_{I}(t)$ 和 $P N_{Q}(t)$ 为扩频 码集中的 PN 码, 且两支路的扩频码是相互独立的。当发 送同步信息时, 两支路同时发送同步扩频码 $P N_{s y n}(t)$, 此时 $P N_{I}(t)=P N_{Q}(t)=P N_{s y n}(t)$ 。

在接收端, 信号经正交下变频和低通滤波后, I、Q 支 路的信号为:

$$
\begin{aligned}
& r_{I}(t)=P N_{I}(t) \cos (\varphi)-P N_{Q}(t) \sin (\varphi) \\
& r_{Q}(t)=P N_{I}(t) \sin (\varphi)+P N_{Q}(t) \cos (\varphi)
\end{aligned}
$$

其中 $\varphi$ 为收发端存在的载波相偏。将本地同步码 $P N_{s y n}(t)$ 分别与 $I 、 Q$ 支路信号进行相关运算。设 $t=t_{0}$ 时, 发 
送端传送同步信息且本地同步码与发送同步码同相，有:

$$
\begin{aligned}
& r_{S I}\left(t_{0}\right)=\int_{t_{0}-T}^{t_{0}} P N_{S y n}(t) r_{I}(t) d(t)=T[\cos (\varphi)-\sin (\varphi)] \\
& r_{S Q}\left(t_{0}\right)=\int_{t_{0}-T}^{t_{0}} P N_{S y n}(t) r_{Q}(t) d(t)=T[\sin (\varphi)+\cos (\varphi)]
\end{aligned}
$$

取两支路信号的平方和得到最大相关峰值:

$$
E_{\max }=r_{S I}\left(t_{0}\right)^{2}+r_{S Q}\left(t_{0}\right)^{2}=2 T^{2}
$$

若同步系统在 $t_{0}$ 时刻输出峰值, 表示该时刻同步帧头 与本地同步码相匹配, 则 $t=t_{0}+k T,(k=0, \pm 1 \ldots)$ 即为同步时 刻。由于同步码是独立于信息码的, 所以在其他时刻, 同 步系统输出的理论值为零。以后第 $k$ 帧的起始时刻为 $t_{k}=t_{0}+k N T$ 。至此, 同步捕获完成, 在此基础上进入同步跟 踪阶段。

实现同步后, 利用同步信息进一步分析载波相偏。将 同步时刻 $I 、 Q$ 支路相关峰输出 $r_{S I}\left(t_{0}\right)$ 和 $r_{S Q}\left(t_{0}\right)$ 写成复数形 式:

$R_{S}\left(t_{0}\right)=r_{S I}\left(t_{0}\right)+j r_{S Q}\left(t_{0}\right)=T(1+j) e^{j \varphi}=\sqrt{2} T e^{j(\varphi+\pi / 4)}=\sqrt{2} T e^{j \varphi^{\prime}}$

其中 $\varphi^{\prime}=\varphi+\pi / 4$ 可以用同步相关峰复数值的实部 和虚部运算得到 $\varphi^{\prime}$ 值, 进一步得到 $\varphi$ 值 ${ }^{[7]}$ 。正交下变频模 块可以根据得到的 $\varphi$ 值进行载波相偏的纠正。

\section{2 同步性能仿真}

由上述分析可知, 本同步模块只有在同步时刻输出峰 值, 理想情况下其他时刻的输出为零。而用文献[7]的方法, 非同步时刻最大值为同步时刻峰值的一半，如图 4 所示。

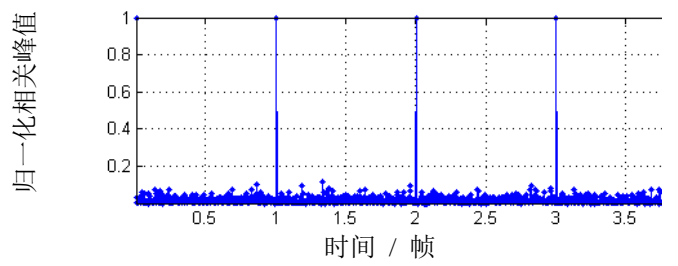

(a) 新系统同步系统峰值

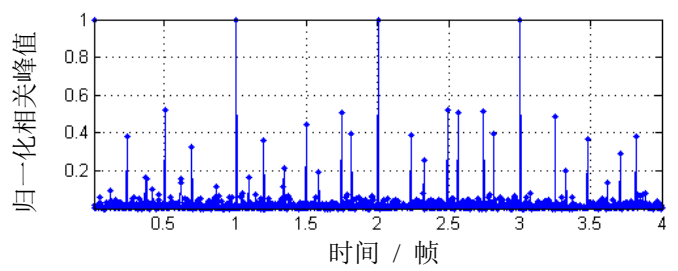

(b) 文献[7]系统同步系统峰值

图 4 同步系统归一化相关峰值图

为了衡量同步相关峰值的明显性, 我们定义参数 $P P D$ (Peak Power Difference):

$$
P P D=10 \log _{10}\left(E_{\max } / E_{2}\right)
$$

其中 $E_{\max }$ 表示一个帧周期内同步系统输出峰值, $E_{2}$ 表 示一个帧周期内同步系统输出值中除了 $E_{\text {max }}$ 外的峰值。新 系统和文献[7]的平均 PPD 参数如图 5 所示, 仿真环境为 MATLAB, 扩频码用 Gold 序列, 扩频码长度为 63 , 每个 扩频码传输 4 比特信息, 帧长为 4 个扩频码长度, $P P D$ 数 值取 1000 个帧周期计算结果的平均值。可见, 本文的同步 方案的性能要明显优于文献[7]。

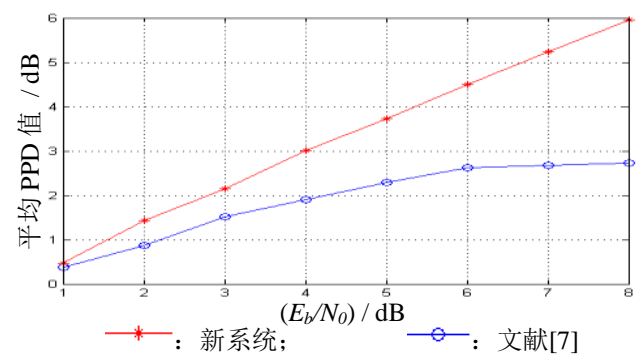

图 5 同步系统输出平均 PPD 参数随信噪比的变化

\section{4. 解扩算法和性能}

\section{1 解扩算法}

在接收端, 系统同步后, 每个支路设置 $U / 2$ 个基于变 换域处理的循环相关器 ${ }^{[4][5]}$ 。在一个扩频码周期内, 第 $i$ 个 相关器计算 $P N_{i}$ 与接收信号的循环相关, 输出为 $V$ 值序列 $\left\{R_{(i, j)} \mid j=1,2 \cdots V\right\}$, 序列元素的序号 $j$ 对应 CPSK 调制的循环 移位值 $v$ 。所有 $U / 2$ 个循环相关器的输出构成一个 $U / 2 \times V$ 的相关矩阵 $\boldsymbol{R}=\left[R_{(i, j)} \mid i=1,2 \cdots U / 2 ; j=1,2 \cdots V\right]$ 。对相关矩阵元 素的绝对值进行择大判决, 最值的正负号和最值的行序号 $i$ 可确定对应的前 $m$ 比特二进制数据, 最值的列序号 $j$ 可确 定对应的后 $n$ 比特二进制数据。解扩系统原理框图如图 6 所示。

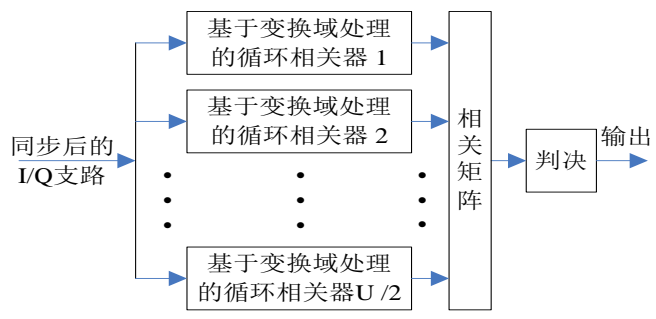

图 6 混合 $\mathrm{M}$ 元扩频解扩原理框图

\section{2 解扩性能分析}

下面分析其误码率性能。无论是正交扩频还是 CPSK 扩频, 其本质都是多元正交信号传输系统。本文所述的混 合 $\mathrm{M}$ 元扩频, 本质上是一个 $\mathrm{M}$ 元双正交信号传输系统。 
在加性高斯白噪声(additional white gauss noise, AWGN)信 道环境下, 根据文献[11], 混合 $\mathrm{M}$ 元扩频通信系统每个支 路的符号错误概率为:

$$
P_{M}=1-\frac{1}{\sqrt{2 \pi}} \int_{-\sqrt{2 E_{s} I N_{0}}}^{\infty}\left(\frac{1}{\sqrt{2 \pi}} \int_{-\left(v+\sqrt{2 E_{s} / N_{0}}\right)}^{v+\sqrt{2 E_{s} / N_{0}}} e^{-\frac{x^{2}}{2}} d x\right)^{\frac{M}{2}-1} e^{-\frac{v^{2}}{2}} d v
$$

其中 $E_{S}$ 为符号能量, $N_{0}$ 为加性高斯白噪声双边功率谱 密度。比特错误概率为:

$$
P_{b}=\frac{2^{k-1}}{2^{k}-1} P_{M}
$$

其中 $k=\log _{2} M$ 。因为 $I 、 Q$ 支路数据信息是平均分配的, 故系统总的比特错误概率也为 $P_{b}$ 。

\section{3 解扩性能仿真}

利用 MATLAB 软件建立混合 M 元扩频通信系统模型, 用 Monte-Carlo 方法进行系统性能仿真。扩频码采用 Gold 序列, 序列长度为 63 。混合 $\mathrm{M}$ 元扩频采用 $k=m+n$ 体制, 即每个扩频码传递 $k$ bit 信息, 其中正交扩频传递 $m$ bit, CPSK 传递 $n$ bit。分别仿真了 $k=3=3+0 、 k=4=3+1 、 k=5=3+2$ 、 $k=6=3+3$ 等几种情况下系统误比特率, 如图 7 所示。

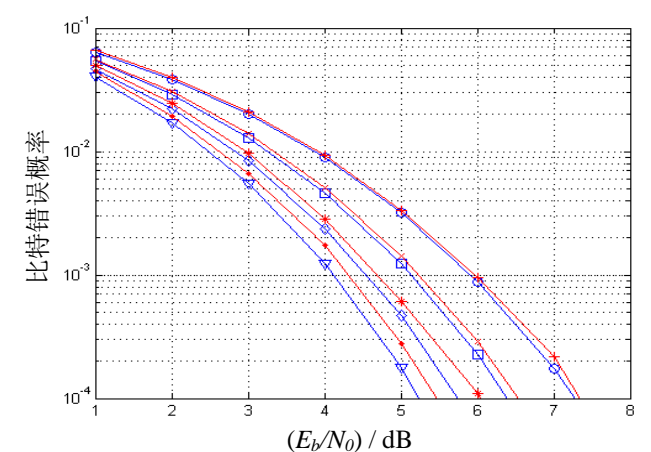

理论值: $-: k=3$; $\square: k=4 ;-\bullet: k=5 ;-\nabla: k=6$; 仿真值: + $-k=3$; $\rightarrow: k=4$; *: $k=5$; $\rightarrow: k=6$;

图 7 新系统误比特率理论值与仿真值

仿真结果表明: 新系统误比特性能理论值与仿真值基 本一致, 验证了系统的正确性。但随着 $k$ 的增大, 系统性 能有少量损失, 主要是由于扩频码的相关性能不理想造成 的。新系统的误比特性能与传统的双正交 M 元扩频一致。 但在扩频增益、信号占用带宽、扩频码条数相同的情况下， 新系统的信息传输速率要大大优于传统的双正交 M 元扩频 和 CPSK 扩频，提高了频带利用率。

\section{5. 结论}

本文提出了一种正交扩频与 CPSK 扩频相结合的高性 能混合 $\mathrm{M}$ 元扩频通信方案。根据理论分析和仿真可知, 与
传统的正交扩频或 CPSK 扩频相比, 该方案具有如下优点: 大大提高了频带利用率, 一个扩频码可以传输 $k=m+n$ 比特 信息，比双正交扩频多 $n$ bit，比 CPSK 多 $m$ bit；扩频码更 具有随机性, 信号频谱更接近高斯白噪声, 故具有更强的 抗截获性; 利用单独的同步码进行同步, 提高了系统同步 性能。该方案的劣势在于增加了系统复杂度。但在一些信 噪比较低、信息传输速率很宝贵的应用场合, 比如深空通 信、卫星隐蔽通信等, 用系统复杂度的增加来换取更好的 性能还是很有必要的。

\section{参考文献(References)}

[1] Chiang C T, "Performance analysis of M-ary DS-CDMA systems with imperfect power control communications". IEE Proceedings, vol. 151, no.6, pp.574-580, 2004.

[2] FU Jiang-zhi, GUO Li-Li, YANG Hong-qiao, "Performances of M-ary orthogonal code spread spectrum system based on composite sequences", Systems Engineering and Electronics, vol. 32, no.4, pp.703-706, 2010.

[3] ZENG Zhi-bin, ZHAUNG Yi-qi, XIANG Xinz, etc. "Scheme of double M-ary spread-spectrum communication based on orthogonal channels", Journal of System Simulation, vol.23, no.5, pp.984-986, 2011.

[4] YAO Jun, CHU Zhen-yong, YI Ke-chu, "Performance analysis of a dual M- ary orthogonal spread spectrum system based on orthogonal cyclic codes", Telecommunication Engineering, vol.46, no.5, pp.84-88, 2006.

[5] Chu Zhen-yong, Ying Xiao-fan, Yi Ke-chu, etc. "An M-ary spread spectrum receiver based on orthogonal cyclic codes," Journal of Electronics \& Information Technology, vol.27, no.11, pp.1704-1709, 2005.

[6] Yindi Yao, Peng Xu, Zhibin Zeng, "Algorithm on improved $\mathrm{M}$-ary spread-spectrum communication”, in 2011 International Conference on Electronic \& Mechanical Engineering and Information Technology, IEEE, 2011, pp.4819- 4822.

[7] ZENG Zhi-bin,ZHUANG Yi-qi1,XIANG Xin, "Double CPSK spread-spectrum communication system based on orthogonal channel," Journal of Sichuan University (Engineering Science Edition),vol.41,no.5,pp.206-210, 2009.

[8] F. Adachi, D. Garg, S. Takaoka,etc, "Broadband CDMA techniques," IEEE Trans.Wireless Commun. vol.12,no.2, pp.8-18, 2005.

[9] Jiang Yimin, Richmod R L, Baras J S. "Carrier frequency estimation of MPSK nodulated signals", in CSHCN, International Conference on Communications, March 1999, Vancouver, Canada, NASA and Hughes Network Systems, pp.1-22.

[10] Soumur E, Bottomley G E. "Effect of frequency offset on DS-SS acquisition in slowly fading channels", in Wireless Communications and Networking Conference, NEW Orleans, USA, IEEE Press, 1999, pp.569-573.

[11] John G. Proakis. Digital comrmunications. 2nd. Beijing: Publishing House of Electronics Industry, 2003:188-191. 